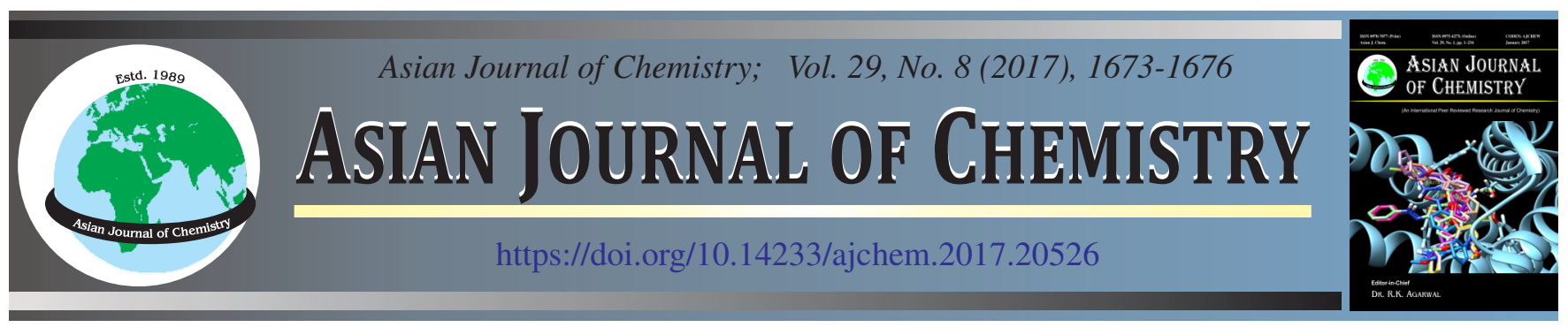

\title{
Synthesis and Characterization of Orange Pigments from Rare Earth Metal Ions
}

\author{
R. Govinda RaO* and D. Divya
}

Department of Chemistry, School of Basic Sciences, Vels University, Pallavaram, Chennai-600 117, India

*Corresponding author: E-mail: govind.sbs@velsuniv.ac.in; govindchem2005@ gmail.com

Keywords: Inorganic pigments, Calcination, Rare earth metal ions, Doping, DRS, Mass tone, Tint strength.

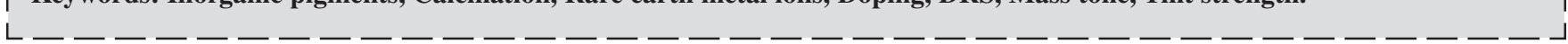

\section{INTRODUCTION}

Colourants are characterized [1-3] by their ability to absorb light selectively in the visible region $(380-780 \mathrm{~nm})$. Electronic (absorption and reflection) spectra have been used to investigate some corrections between chemical structure and colour of the species. The characteristic parameters of the spectrum, i.e. the positions of absorption bands and their intensity and shape are important factors in the determination and prediction of colour [4] and these parameters are dependent upon the electronic structure and symmetry of the clues. Theoretical problems connected with the nature of colour have been studied extensively, especially in case of the organic dyes.

One of the most important problems in colour technology is how to attain a specified colour using a mixture of colourants [5]. A tristimulus colorimetry is generally employed for colour measurement. The principle of operation involves projection of light of a known spectral energy on the pellet and measurement of the intensity of the reflected light using photo detectors. All the synthesized pigment samples are found to be having colour coordinates and exhibit the colour from pale yellow to orange red. Reflectance spectra of the samples show high reflectance percentage in the $600-800 \mathrm{~nm}$ range. Because of the homogeneity of the phase formed the intense colouration the yttrium doped pigments have been found to be superior to cerium doped pigments as possible ecological inorganic pigments.
Colour arises from the way in which colourants interact with light. The part which has extended delocalized system of electrons called a chromophore. A sequence of alternating double bonds through which the electrons are spread is termed a conjugated system [6-8]. Group of atoms which can modify the colour are called auxochromes. These groups contain lone pair of electrons that become part of the delocalized electron system. Coloured inorganic compounds often contain transition metals in which the $d$ subshell of electrons is split by attached groups of the ligands. The extent of this splitting is responsible for the colour.

Here, in this study we have prepared new inorganic pigments through calcination and characterized by infrared, UV-visible spectroscopy and diffuse reflectance spectroscopy (DRS). The activities of pigments were used to study mass tone and tint strength.

\section{EXPERIMENTAL}

Lanthanum chloride, lanthanum acetate, cerium carbonate, cerium chloride, cerium acetate and cerium sulfate were obtained from M/s Sigma Aldrich Ltd., UK.

The rare earth salts were mixed in varying stoichiometric ratio with salts of transition metal ions, either individually or in combination in an agate mortar with a pestle. The mixture was calcinated at a temperature of $800{ }^{\circ} \mathrm{C}$ for $2 \mathrm{~h}$ in air.

General procedure: The calcinations was carried out in a small electrically heated, fiber insulated high temperature 
furnace with extremely short heating and cooling cycles, which had an useful chamber dimensions: app. $150 \mathrm{~mm} \times 150 \mathrm{~mm} \times$ $200 \mathrm{~mm}(\mathrm{w} \times \mathrm{h} \times \mathrm{d})$.

The chamber volume of the furnace was approximately 4 L. the external dimension of the furnace was approximately $600 \mathrm{~mm} \times 600 \mathrm{~mm} \times 600 \mathrm{~mm}(\mathrm{w} \times \mathrm{h} \times \mathrm{d})$. It had a double wall high quality SS/MS construction and was poser coated. A stainless steel door with proper insulation was provided for sample placement.

The construction of the furnace was based on high alumina A32 insulation with a backup of zirconia blend ceramic fiber. The skin temperature was maintained at ambient by air cooling. The maximum operating temperature was $1600{ }^{\circ} \mathrm{C}$ in oxidizing atmosphere.

The heating rate was variable form 1 to $20^{\circ} \mathrm{C} / \mathrm{min}$ with an accuracy of $\pm 1{ }^{\circ} \mathrm{C}$. The heating was through Kanthal-Super1700 6/12 heating elements. It had a Pt + Rh30 \%/Pt + Rh6 \% - B type thermocouple and Eurotherm PID digital temperature controller programmable with programs/16 segments for appropriate programming.

Detection method: The obtained powders were characterized by diffuse reflectance. UV-visible spectra were recorded using a Perkin Elmer Lambda 35 UV-visible spectrophotometer equipped with Lab sphere RSA-PE-20 diffuse reflectance accessory.

Barium sulfate was used as a white standard. The principle of operation involved projection of a light of a known spectral energy on the pellet of pigment kept at $90^{\circ}$ to the light source and measurement of the intensity of the reflected light using photodetectors.

CIE illuminant D65 was used in all the colour measurements. The CIELab (1976) colour space was used for colour measurement; the colourimetric values were computed using advanced spectroscopy software (Perkin Elmer).

The band gap values were calculated using Kubelk Munk function which is given by $K-M=(1-R)^{2} / 2 R$, where $R$ is the reflectance value. A plot of K-M and wavelength is drawn and the absorption edge is determined from the plot. The value of the wavelength is substituted in the formula $\mathrm{E}(\mathrm{eV})=1236$ / wavelength $(\mathrm{nm})$.

The hue angle $h$ (Fig. 1) is defined as starting at $+a^{*}$ axis and is expressed in degrees. An h value of $90^{\circ}$ would indicate yellow colour. Larger $\mathrm{C}^{*}$ values indicate a higher Chroma or saturation. Chroma can also be referred to as cleanliness of the colour.

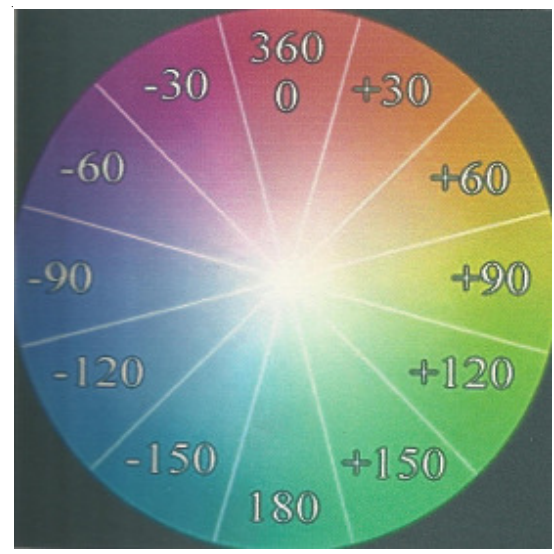

Fig. 1. Hue angle and colour

\section{RESULTS AND DISCUSSION}

Lanthanum based pigments: Formulations were developed with varying lanthanum and first row transition metal ions viz., chromium, manganese and iron. Lanthanum chloride salt was employed in the study. The ratio of $\mathrm{La}$ to $\mathrm{Cr} / \mathrm{Mn} / \mathrm{Fe}$ was 1:0:1. Increase the temperature of $800{ }^{\circ} \mathrm{C}$ at a rate of $5^{\circ} \mathrm{C} / \mathrm{min}$. The maximum reflectance for the green pigment was around $550 \mathrm{~nm}$ and that for iron pigment was around $700 \mathrm{~nm}$ (Fig. 2). Accordingly the CIELAB colour coordinates varied and are presented (Table-1). The $\mathrm{Cr}$ and $\mathrm{Mn}$ based pigments were of greenish-yellow and that of Fe was more in the orange side $[9,10]$. In the absence of lanthanum, the purer oxides of chromium, manganese and iron were formed. There was significant change in the $\mathrm{a}^{*}$ and $\mathrm{b}^{*}$ values in the pigments formed with $\mathrm{Cr}$ and $\mathrm{Fe}$, while no such change was observed with Mn.

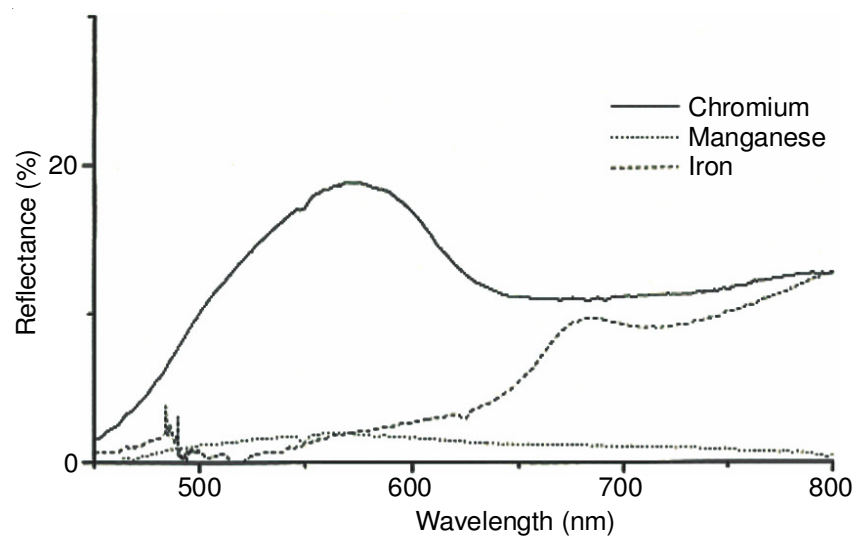

Fig. 2. Reflectant spectra of $\mathrm{La}-\mathrm{Cr} / \mathrm{Mn} / \mathrm{Fe}$ pigment

\begin{tabular}{lccccc}
\multicolumn{7}{c}{ TABLE-1 } \\
\multicolumn{7}{c}{ COLOUR COORDINATES FOR La-Cr/Mn/Fe PIGMENTS } \\
\hline Metal ions & $\mathrm{L}$ & $\mathrm{a}^{*}$ & $\mathrm{~b}^{*}$ & $\mathrm{~h}^{\circ}$ & $\mathrm{C}$ \\
\hline $\mathrm{Cr}$ & 0.30 & 0.01 & 0.49 & 89.4 & 0.50 \\
$\mathrm{La}: \mathrm{Cr}$ & 46.7 & -6.0 & 42.5 & 98.1 & 42.9 \\
$\mathrm{Mn}$ & 43.0 & 1.4 & 15.9 & 85.0 & 16.0 \\
$\mathrm{La}: \mathrm{Mn}$ & 13.2 & -2.9 & 18.0 & 99.1 & 38.8 \\
$\mathrm{Fe}$ & 3.6 & 13.9 & 6.3 & 24.4 & 15.3 \\
$\mathrm{La}: \mathrm{Fe}$ & 28.7 & 18.0 & 34.4 & 62.4 & 38.8 \\
\hline
\end{tabular}

Role of associated ion in the colour of pigment: When the chloride ion in lanthanum was replaced with acetate or carbonate, no significant change in the colour. In the pigments, when chloride ion was replaced with acetate and carbonate for a La: Fe Pigment [11], the bandgap energy (eV) changed from 2.103 to 2.39 and 2.47 respectively (Fig. 3). An increase in the bandgap energy $(\mathrm{eV})$ by 0.1 units as the anion was replaced is indicative of the change in the colour of the pigment. This is possible as acetate or carbonate would probably be lost completely as $\mathrm{CO}_{2}$ while a chloride ion would possibly be retained in the system.

Cerium based pigment: Pigments were prepared from a homogeneous mixture of cerium carbonate and chromium/ manganese/iron/molybdenum. The raw materials were calcinated at $800{ }^{\circ} \mathrm{C}$ for a period of $120 \mathrm{~min}$. The chromium based pigment had very characteristic reflectance maxima at 600 nm (Fig. 4). In all the cases the hue angle varied by only $10^{\circ}$ in the presence/absence of cerium. It can be seen that $\mathrm{Ce}$ and 


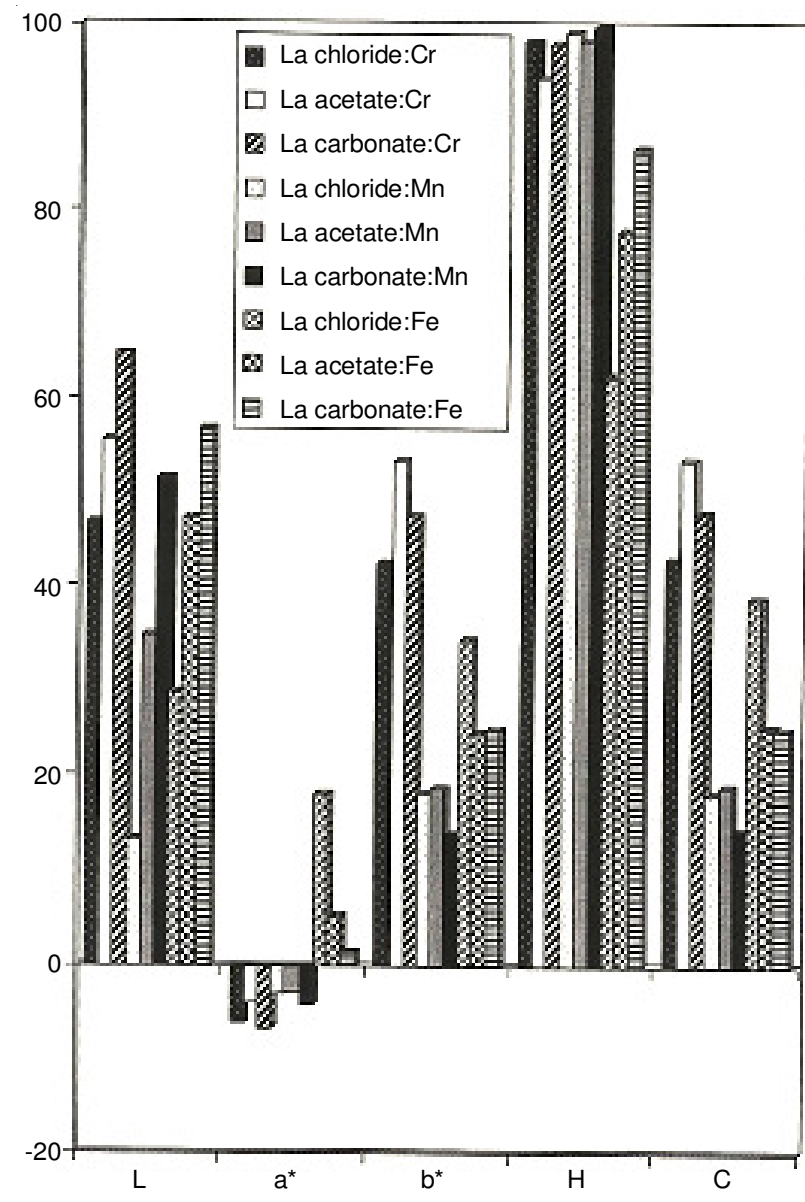

Fig. 3. Variation in colour coordinates with chloride, acetate and carbonate of lanthanum

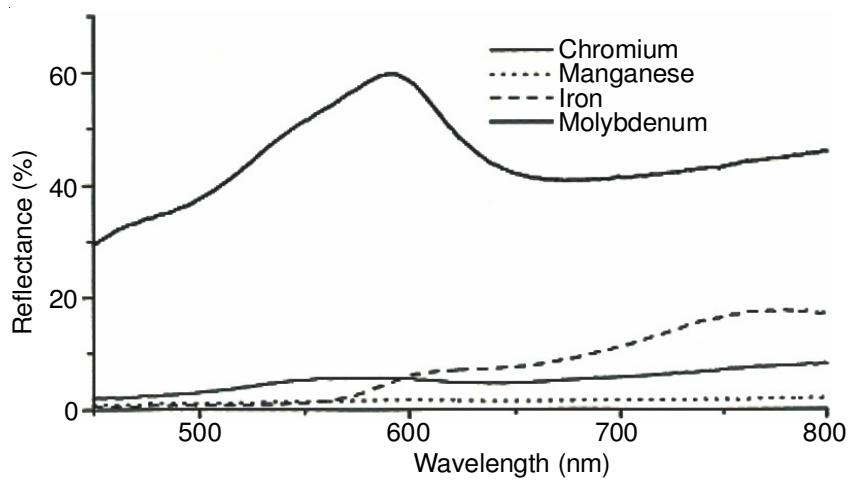

Fig. 4. Reflectance spectra of Ce:Cr, Ce:Mn, Ce:Fe and Ce:Mo

La combined with dopants have a characteristic reflectance at around $600 \mathrm{~nm}$, which can be attributed to the role of the rare earth ion (Table-2).

TABLE-2

COLOUR COORDINATES FOR Ce-Cr/Mn/Fe PIGMENTS

\begin{tabular}{lccccc}
\hline Metal ions & $\mathrm{L}$ & $\mathrm{a}^{*}$ & $\mathrm{~b}^{*}$ & $\mathrm{~h}^{\circ}$ & $\mathrm{C}$ \\
\hline $\mathrm{Cr}$ & 0.30 & 0.01 & 0.49 & 89.4 & 0.50 \\
$\mathrm{Ce}: \mathrm{Cr}$ & 26.8 & 0.6 & 15.6 & 87.9 & 15.6 \\
$\mathrm{Mn}$ & 43.0 & 1.4 & 15.9 & 85.0 & 16.0 \\
$\mathrm{Ce}: \mathrm{Mn}$ & 12.6 & 2.0 & 7.8 & 75.5 & 8.1 \\
$\mathrm{Fe}$ & 3.6 & 13.9 & 6.3 & 24.4 & 15.3 \\
$\mathrm{Ce}: \mathrm{Fe}$ & 21.5 & 23.6 & 28.1 & 50.0 & 36.7 \\
Mo & 34.5 & 12.5 & 41.2 & 73.2 & 43.1 \\
Ce:Mo & 76.9 & 0.2 & 21.9 & 89.4 & 21.9 \\
\hline
\end{tabular}

Role of cerium salt in colour of the cerium pigment: When cerium carbonate, cerium sulfate, cerium oxalate, cerium acetate and cerium chloride were doped with ferrous sulfate [12] such that the Ce:Fe ratio was 1: 0.1 (Table-3), the band gap energy varied as 2.08, 2.08, 2.7, 2.1 and 2.06 respectively (Fig. 5). A major shift in the band gap energy (eV) was observed only with cerium oxalate.

\begin{tabular}{lccccc}
\multicolumn{7}{c}{ TABLE-3 } \\
\multicolumn{7}{c}{ COLOUR COORDINATES FOR Ce-Fe PIGMENTS } \\
\hline Cerium salt & $\mathrm{L}$ & $\mathrm{a}^{*}$ & $\mathrm{~b}^{*}$ & $\mathrm{~h}^{\circ}$ & $\mathrm{C}$ \\
\hline Carbonate & 21.5 & 23.5 & 28.1 & 50.0 & 36.7 \\
Sulfate & 60.5 & 20.3 & 30.7 & 56.6 & 36.8 \\
Oxalate & 59.8 & 4.9 & 35.5 & 82.2 & 35.9 \\
Acetate & 55.4 & 14.7 & 18.2 & 51.0 & 23.4 \\
Chloride & 44.8 & 20.8 & 25.7 & 51.0 & 33.0 \\
\hline
\end{tabular}
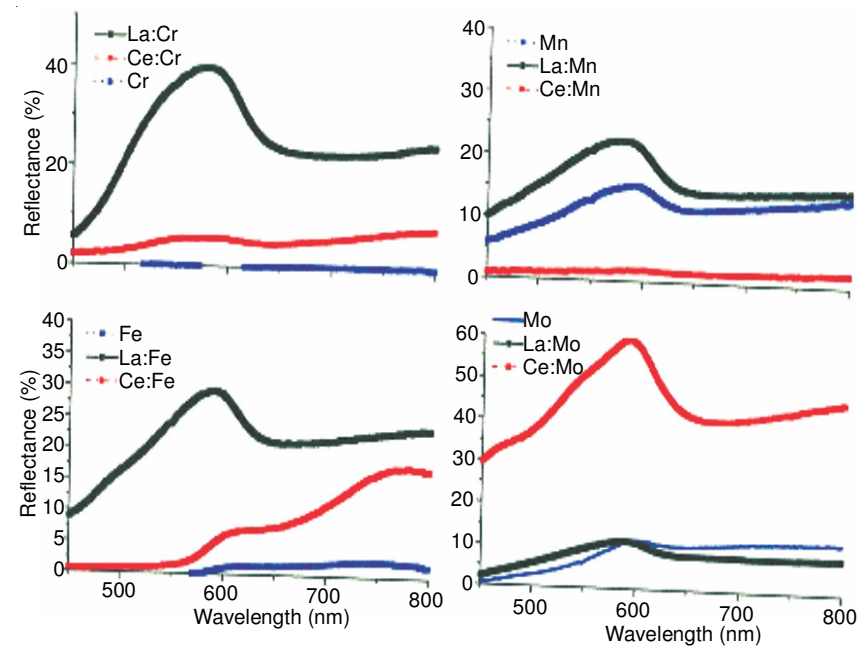

Fig. 5. Reflectance spectra of dopants $\mathrm{La} / \mathrm{Ce}: \mathrm{Cr}, \mathrm{La} / \mathrm{Ce}: \mathrm{Mn}, \mathrm{La} / \mathrm{Ce}: \mathrm{Fe}, \mathrm{La} /$ Ce:Mo

Role of cerium:metal ion ratio in colour of the cerium pigment: When the cerium(III) chloride was maintained constant and the ratio of dopant varied such the resultant ratio was 10:1 to $10: 5$. The colour of the product is also changed as expected. There was no major change in the reflectance spectra, indicating that the colour remained more or less same. However, the intensity of the colour as reflected by a lower reflectance or in turn higher absorbance was seen as the dopant $(\mathrm{Cr})$ concentration increased (Fig. 6).

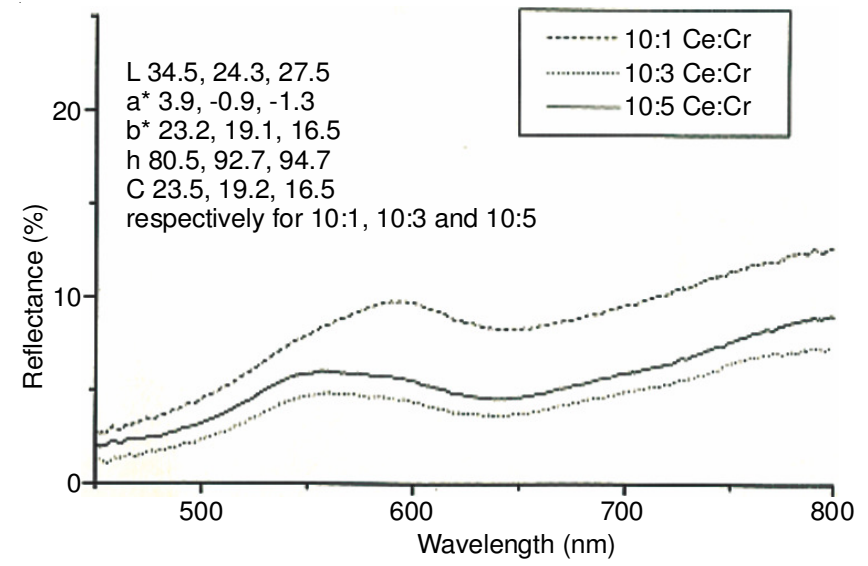

Fig. 6. Reflectance spectra of $\mathrm{Ce}: \mathrm{Cr}$ at varying ratio 
Role of multiple doping in colour of the cerium pigment: When a single dopant was used the colour of the resulting pigment either reflected the colour of the dopant of that of cerium oxide. Multiple doping resulted in higher bandgap energy and lower L values (Table-4), indicating that the pigments had a much darker shade. The characteristic reflectance maxima were observed around $600 \mathrm{~nm}$ (Fig. 7). In the case of Ce:Mo:Fe [13], this reflectance maxima was very much pronounced and this corresponded to a bright orange colour, which was different from that of the individual dopants either alone or in combination (in the absence of cerium).

\begin{tabular}{lcccccc}
\multicolumn{7}{c}{ TABLE-4 } \\
\multicolumn{7}{c}{$\begin{array}{c}\text { COLOUR COORDINATES FOR Ce-M } \\
\text { PIGMENTS AT VARYING RATIOS }\end{array}$} \\
\hline \multicolumn{1}{c}{ Cerium salt } & $\mathrm{L}$ & $\mathrm{a}^{*}$ & $\mathrm{~b} \mathrm{~b}^{*}$ & $\mathrm{~h}^{\circ}$ & $\mathrm{C}$ & $\begin{array}{c}\text { Band- } \\
\text { gap } \\
\end{array}$ \\
& & & & & & $(\mathrm{eV})$ \\
\hline $\mathrm{M}_{1}-\mathrm{Cr}, \mathrm{M}_{2}-\mathrm{Mo}$ & 27.7 & 7.1 & 16.4 & 66.6 & 17.9 & 2.2 \\
$\mathrm{M}_{1}-\mathrm{Cr}, \mathrm{M}_{2}-\mathrm{Mn}$ & 30.9 & 3.2 & 23.3 & 82.2 & 23.5 & 2.3 \\
$\mathrm{M}_{1}-\mathrm{Cr}, \mathrm{M}_{2}-\mathrm{Fe}$ & 19.3 & 5.5 & 16.5 & 71.7 & 17.3 & 2.2 \\
$\mathrm{M}_{1}-\mathrm{Mn}, \mathrm{M}_{2}-\mathrm{Mo}$ & 48.6 & 9.7 & 25.2 & 68.9 & 26.9 & 2.1 \\
$\mathrm{M}_{1}-\mathrm{Mn}, \mathrm{M}_{2}-\mathrm{Fe}$ & 38.2 & 1.2 & 9.7 & 82.9 & 9.8 & 2.2 \\
$\mathrm{M}_{1}-\mathrm{Fe}, \mathrm{M}_{2}-\mathrm{Mo}$ & 40.1 & 12.0 & 44.2 & 74.9 & 45.7 & 2.3 \\
\hline
\end{tabular}

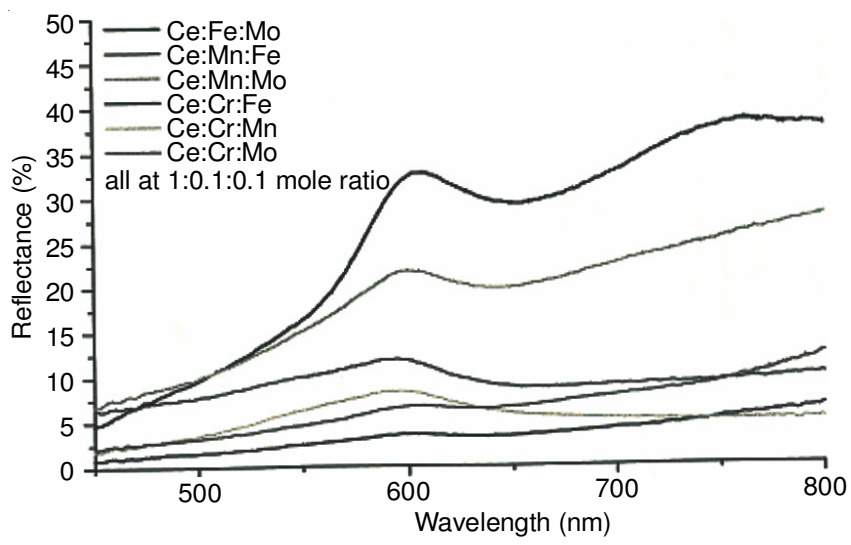

Fig. 7. Reflectance spectra of Ce:M1:M2 at 1.0:0.1:0.1 ratio

Development of paint and analysis of mass tone and tint strength: The developed pigment with combination of Ce:Mo:Fe in the ratio 1.0:0.1:.01 was prepared in bulk at 100 g quantities $[14,15]$. The paint thus developed was coated on a checkered board having a white and black background. The ability of the pigment to cover the black reflected its mass tone or hiding power (Fig. 8). The ability of the pigment to retain its colour when mixed with 5 parts of titanium dioxide for every part of the pigment corresponded to the tint strength of the pigment.

\section{Conclusion}

A replacement for cadmium, cobalt, lead and mercury based pigments has been sought through the use of rare earth metal ions - cerium and lanthanum. Solid state reactions of these metal ions with transition metal ions like chromium, molybdenum, manganese and iron were carried out individually. The reflectance spectra, band gap energy (eV), CIELAB colour coordinates were measured and the colour of the developed pigments characterized. It was found that cerium offered better scope for doping than lanthanum as it produced

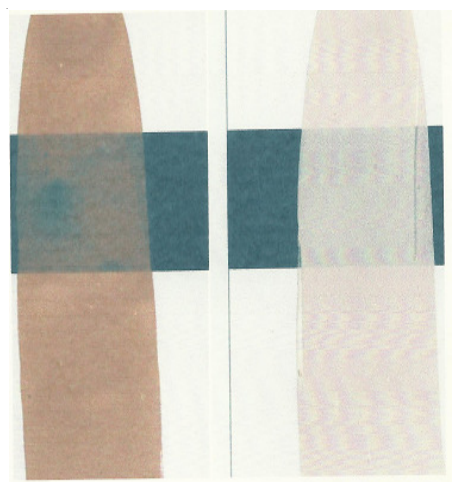

Fig. 8. Mass tone (left) and tint strength (right) of developed Ce-Mo-Fe pigment

better shades. The associated ions did not have any significant influence on the colour of the pigment i.e. a cerium(III) chloride behaved more or less similar to that of cerium(III) carbonate and cerium(III) acetate. Individual doping resulted in colours, which were closer to either that of cerium oxide or the dopant oxide.

Multiple doping experiments revealed interesting results: An optimal doping of $\mathrm{Ce}: \mathrm{Mn}: \mathrm{Fe}$ and Ce:Mo:Fe resulted in reflectance maxima at $600 \mathrm{~nm}$, closer to a red shade. The best results were obtained for Ce:Mo:Fe at a ratio of 1:.01:0.1 and a treatment temperature of $800{ }^{\circ} \mathrm{C}$ and duration of treatment of $120 \mathrm{~min}$. A paint developed for the pigment had good mass tone and covering poser and a reasonable tint strength.

\section{REFERENCES}

1. A. Roy, Artists' Pigments: A Handbook of Their History and Characteristics, National Gallery of Art, Washington, DC, vol. 2 (1993).

2. D. Bomford, J. Kirby, J. Leighton and A. Roy, Art in the Making: Impressionism, Yale University Press, New Haven, CT (1990).

3. J.A. Badenes, M. Llusar, M.A. Tena, J. Calbo and G. Monrós, J. Eur. Ceram. Soc., 22, 1981 (2002); https://doi.org/10.1016/S0955-2219(01)00517-9.

4. P. Ball, Bright Earth: Art and the Invention of Color. Farrar, Straus and Giroux (2002).

5. M. Doerner, The Materials of the Artist and Their Use in Painting: With Notes on the Techniques of the Old Masters, Revised Edition (1984).

6. V. Finlay, Color: A Natural History of the Palette, Random House (2003).

7. S. Furukawa, T. Masui and N. Imanaka, J. Alloys Comp., 418, 255 (2006);

https://doi.org/10.1016/j.jallcom.2005.08.108.

8. R.L. Feller, Artists' Pigments: A Handbook of Their History and Characteristics, National Gallery of Art, Washington, DC, vol. 1 (1986).

9. E.W. Fitzhugh, Artists' Pigments: A Handbook of Their History and Characteristics, National Gallery of Art, Washington, DC, vol. 3 (1997).

10. J. Gage, Color and Culture: Practice and Meaning from Antiquity to Abstraction, University of California Press (1999).

11. D. Giaume, V. Buissette, K. Lahlil, T. Gacoin, J.-P. Boilot, D. Casanova, E. Beaurepaire, M.-P. Sauviat and A. Alexandrou, Prog. Solid State Chem., 33, 99 (2005); https://doi.org/10.1016/j.progsolidstchem.2005.11.041.

12. B. Gonzalvo, J. Romero, F. Fernández and M.J. Torralvo, J. Alloys Comp., 323-324, 372 (2001); https://doi.org/10.1016/S0925-8388(01)01056-8.

13. M. Jansen and H.P. Letschert, Nature, 404, 980 (2000); https://doi.org/10.1038/35010082.

14. L.S. Kumari, P.P. Rao, S. Sameera and P. Koshy, Ceram. Int., 38, 4009 (2012);

https://doi.org/10.1016/j.ceramint.2012.01.057.

15. R. Meyer, The Artist's Handbook of Materials and Techniques, Viking, edn 5 (1991). 